\title{
Autonomic dysfunctions in multiple sclerosis: Challenges of clinical practice (Review)
}

\author{
CARMEN ADELLA SIRBU ${ }^{1,2 *}$, RALUCA-MIHAELA MEZEI ${ }^{2}$, \\ CRISTIAN FALUP-PECURARIU ${ }^{3,4}$, OVIDIU GABRIEL BRATU ${ }^{5,6}$, ANCA MARIA SIRBU ${ }^{7 *}$, \\ MINERVA CLAUDIA GHINESCU ${ }^{1 *}$ and FLORENTINA IONITA RADU ${ }^{1,8}$ \\ ${ }^{1}$ Department of Preclinical Disciplines, Faculty of Medicine, 'Titu Maiorescu' University, \\ 031593 Bucharest; ${ }^{2}$ Department of Neurology, Central Military Emergency University Hospital, 010242 Bucharest; \\ ${ }^{3}$ Department of Neurology, County Emergency Clinic Hospital, 500365 Brasov; ${ }^{4}$ Department of Neurology, \\ Faculty of Medicine, Transilvania University, 500036 Braşov; ${ }^{5}$ Department of Urology, 'Carol Davila' University \\ of Medicine and Pharmacy, 050474 Bucharest; ${ }^{6}$ Department of Urology, Central Military Emergency University Hospital, \\ 010242 Bucharest; ${ }^{7}$ Department of Endocrinology, 'C. I. Parhon' National Institute of Endocrinology, 011863 Bucharest; \\ ${ }^{8}$ Department of Gastroenterology, Central Military Emergency University Hospital, 010242 Bucharest, Romania
}

Received July 10, 2020; Accepted August 11, 2020

DOI: $10.3892 /$ etm.2020.9326

\begin{abstract}
Multiple sclerosis, demyelinating, inflammatory, degenerative, and chronic disease, raises many challenges in terms of disease management. The autonomic nervous system is affected by neuroinflammation but also contributes to its maintenance and the evolution of the disease. Multiple sclerosis interfering with parasympathetic or sympathetic modulation may influence the immune response. Less attention is paid to autonomic dysfunctions, although they produce a serious impact on the quality of life. In addition to motor disabilities, patients also have non-motor dysfunctions. Regardless of its clinical forms, patients with multiple sclerosis may have autonomous disturbances such as bladder, sexual, cardiovascular, thermoregulatory, gastrointestinal dysfunction and fatigue. These must be identified based on medical history, clinical symptoms, and specific paraclinical tests. In addition to the multitude of immunomodulatory therapeutic agents that influence the progression of the disease, the therapy of autonomic dysfunctions remains difficult to address. However, their identification and treatment lead to increased quality of patient management and avoid complications of this disease.
\end{abstract}

Correspondence to: Professor Cristian Falup-Pecurariu, Department of Neurology, Faculty of Medicine, Transilvania University, 29 Bulevardul Eroilor, 500036 Brașov, Romania

E-mail: crisfp100@yahoo.co.uk

*Contributed equally

Key words: multiple sclerosis, bladder dysfunction, sexual dysfunction, gastrointestinal dysfunction, cardiovascular dysfunction, fatigue, thermoregulatory dysfunction, autonomic nervous system

\section{Contents}

1. Introduction

2. Epidemiology of autonomic dysfunctions in multiple sclerosis

3. Autonomic dysfunctions in MS patients

4. Conclusions

\section{Introduction}

Multiple sclerosis (MS), affects both somatic and autonomic nervous system with a wide range of symptoms and signs. Less attention is paying to autonomic dysfunction, although they produce a serious impact on the patient management. Autonomic dysfunctions in MS patients are the result of multiple factors, such as demyelination of particular structures, imbalance due to the interaction of a dysregulated immune system and specific lymphocyte receptors, vitamin D deficiency and Epstein-Barr virus infection (1). Autonomic dysfunctions may be encountered in different stages of MS patients, diagnosis, and treatment being fundamental. This review aims to present the most common autonomic dysfunctions in patients with multiple sclerosis, providing a much wider view of this particular problem, and highlighting the importance of diagnosis and treatment.

\section{Epidemiology of autonomic dysfunctions in multiple sclerosis}

Autonomic dysfunctions encountered in patients with multiple sclerosis are found in $45-85 \%$ and include bladder, sexual, gastrointestinal, cardiovascular, and thermoregulatory dysfunctions (1). The most common is the urinary bladder dysfunction, its prevalence varying from 30 to $75 \%$, in later stages reaching almost $80 \%$ (2). The most common symptom among male patients is erectile dysfunction, its prevalence being $\sim 60 \%$, with 
an increased prevalence of $>70 \%$ in later stages $(1,2)$. Vaginal dryness and sensory disturbances are the most common complaints among females. Almost $60 \%$ of the multiple sclerosis women encounter sexual dysfunctions. In terms of gastrointestinal dysfunctions, the prevalence varies from 45 to $68 \%$ (1). The cardiovascular disorders are reported to affect $\sim 8-50 \%$ of the patients, implied in both the sympathetic and parasympathetic functions and being related partially to the lesions encountered. Thermoregulation and its dysfunction have not been frequently, reported although heat sensitivity occurs in $60-90 \%$ of the cases (3). Whilst not that often, some patients complain about cold sensitivity, with an incidence of $15 \%$ (3).

\section{Autonomic dysfunctions in MS patients}

Bladder dysfunction, is known to affect $\sim 80 \%$ of the patients, and found mainly in the progressive types rather than remitting ones (2). The first mechanism incriminated is the detrusor-sphincter dissynergy, followed by the detrusor overactivity, both of them leading to urinary retention and in time followed by upper urinary tract dilation which may lead to chronic renal failure and also urinary tract infections. Patients are describing an increased urinary frequency, nocturia, the sensation of urgent voiding, and urinary incontinence (1). The interrupted connection between the sacral micturition and the pontine centers, the lesions in the cervical and thoracic cord, are some explanations (2). Studies show a weak correlation between the site of the lesions and the feature of MRI lesions (2). These findings may contribute to explaining why the bladder dysfunction can be found even from the first years of the disease and it may be the result of the interaction between the overactivity of the immune system and the autonomic nervous system (4). Renal function in patients with MS can be assessed using different methods. To evaluate urinary retention video-guided urodynamic examination can be used, it is the most accurate in determining the bladder function, monitoring urinary flow, the internal pressure of the bladder, and the pelvic muscle activity. While it may be useful in determining the residual urine, ultrasound does not identify the cause and the character of the disturbance that led to it, although it is helpful for therapeutic control. Moreover, the degree of deterioration of the pudendal nerve is useful to test the somatosensory evoked potential (2). Sphincter electromyography may also be helpful, although it is not a standard procedure. The treatment has two targets, one is the inhibition of bladder evacuation and the second is the sphincter relaxation. The most commonly used drugs are anti-cholinergic, cholinergic, and alpha-adrenergic blockers depending on each case. To inhibit the detrusor hyperactivity, parasympathetic suppressor drugs are used. Oxybutynin is the most frequently used, sometimes being also useful for the local injection of botulinum toxin. Also, for mild urge incontinence tricyclic antidepressants can have a beneficial effect (5). Despite the medication, the formation of residual urine and incontinence cannot always be prevented, so sometimes intermittent self-catheterism may be needed, a permanent catheter being the second choice $(6,7)$. Auxiliary to the pharmacological treatment, muscular training of the pelvic floor is also advised. With age, other causes of urinary dysfunction may be added (8).
Sexual dysfunctions are a very common feature among MS patients, some studies associating them with the severity of the disease and with MS type, being more likely to occur in relapsing-remitting clinical forms, others showing no correlation between them (2). While sexual function is influenced by multiple factors, the dysfunction has a multifactorial etiology. Sexual dysfunction is correlated with autonomic and somatic disturbances, with psychological factors, some studies finding associations with depression. Furthermore, it correlates positively with the number of lesions on MRI and with low quality of life. Sexual dysfunctions in male patients are strongly associated with bladder dysfunctions $(1,2)$. Studying the symptoms, the most common is erectile dysfunction, followed by the ejaculation dysfunction, the parasympathetic system is involved in erection whereas sympathetic system being responsible for the ejaculation (1). Female patients are also affected, frequently experiencing anorgasmia, decreased lubrication, lower libido, and sensory dysfunctions in the genital region, although they are not correlated with bladder dysfunction (2). Regarding sexual dysfunctions, diagnostic procedures and autonomic testing methodologies are not specific, but patients are often having abnormal sympathetic skin responses in the genital area that can be confirmed also using the somatosensory evoked potentials for the pudendal nerve. Treatment of this dysfunction in patients with MS is still a challenge. The most commonly used medications are phosphodiesterase 5 inhibitors (PDE-5 inhibitors) for erectile dysfunction and hormonal therapy along with a topical application of estrogen for improving the lubrication in women (1). In the case of contraindication of the PDE-5 inhibitors, a medicated urethral system for erection can be used.

Gastrointestinal dysfunction. Bowel disturbances among patients with MS is also a major problem, being responsible for the poor quality of life and involving both upper and lower gastrointestinal tract (9). The mechanisms involved in gastrointestinal dysfunctions include mainly deterioration of the pyramidal tract and secondary immobility, other factors such as abdominal muscle paresis and hypertonic pelvic muscles are also mentioned in the literature (2). Symptoms such as diarrhea and constipation are mostly the result of the autonomic dysfunctions rather than central nervous system lesions, while the autonomic activity cannot be held responsible for the fecal incontinence which is another common symptom among multiple sclerosis patients (9). Similarly to the bladder dysfunctions, the bowel disturbances are substantially frequent in the progressive type of MS, being positively correlated with the urinary symptoms. Bowel dysfunction may be taken into consideration when patients complain about constipation and diarrhea, these two being the most common manifestations, while fecal incontinence is reported to appear mostly along with its progression (10). Studies show that people complaining about constipation have also a delayed gastric emptying time and it is not uncommon for them to experience upper gastrointestinal symptoms such as nausea, vomiting, and gastroesophageal reflux. Anal manometry, colonic transit time, and the external anal sphincter examination by electromyography, can all confirm the disturbances of the lower gastrointestinal dysfunctions, while for the upper gastrointestinal dysfunctions there are no specific tests. The therapy 
for the gastrointestinal disturbances is aimed to improve the symptoms rather than being addressed to the mechanisms that induced them. Medication includes osmotic and stimulant laxatives, stool softeners, and bulk drugs. Dietary changes and adequate fluid intake are also useful along with conservative management such as physical activity, studies suggesting that the autonomic modulation may represent a future target $(1,10)$.

Cardiovascular dysfunction affects a great number of patients with MS, having a great impact on their quality of life, and involving disturbances in both sympathetic and parasympathetic components (11). The components seem to be affected differently during the evolution of the disease, studies showing that the parasympathetic dysfunction was associated to the scores on the Expanded Disability Status Scale and in conclusion is frequently found in the later stages, while the sympathetic dysfunction was correlated with the inflammatory mechanisms that are involved mainly in the early stages. The fundamental cause of cardiovascular dysfunction is not clearly understood, but they are thought to imply lesions especially in the midbrain, in the limbic structures, insula, or parietal lobe $(1,11)$. Whilst the lesions cannot be considered solely responsible for the cardiovascular disturbances, studies are taking into consideration, as potential causes, the catecholamines released due to the inflammatory lesions in the brain and spinal cord and also autonomic dysregulation. Aluminum has been identified in both intracellular and extracellular brain locations, suggesting a role in neurodegeneration (12). The cardiovascular dysfunction involves reduced heart rate variability and vasomotor disturbances leading to symptoms such as orthostatic intolerance, dizziness, and fatigue, the latter being one of the most common complaints of MS patients (11). These symptoms were reported in several studies, which assessed the variability of the blood pressure and the heart rate responses in both healthy patients and MS, finding abnormal cardiovascular responses in the affected individuals (13-15). Some studies suggest that during the first years after the disease onset, patients with MS are at higher risk of cardiovascular diseases than the general population, but cardiovascular emergencies are rarely acknowledged as prime manifestation of MS. However, a case study reporting a patient that turned out to have MS while presenting to the emergency department with typical signs for Tako-Tsubo cardiomyopathy and nonspecific symptoms for MS such as headache, diaphoresis, tachycardia, and dyspnea alerts us that cardiovascular emergencies should also be taken into consideration (16). One of the easiest methods to evaluate the orthostatic intolerance, that many patients are complaining of, is the Tilt test. To evaluate the sympathetic system Grip test can be performed, some studies finding it to be the most accurate in determining the sympathetic dysfunction. Another way to assess the sympathetic disturbances is through Schellong's test, measuring the blood pressure and heart rate in a supine position and after standing up. For parasympathetic function, breath test and Valsalva's maneuver are the most used, both of them evaluating the changes in the heart rate, some studies finding significant differences between active and inactive disease. Also, the heart rate variability may be evaluated by deep breathing and hyperventilation.

Fatigue. Special attention should be given to fatigue, with a strong correlation with the autonomic dysfunctions, the symptoms are related with the parasympathetic and the sympathetic dysfunctions $(11,17)$. Studies suggest that a decreased vagal function and a normal to low sympathetic activity are related to the perception of fatigue (1). Furthermore, it is well known that patients with MS are complaining about fatigue from the early stages of the disease, which may suggest a common mechanism with the autonomic disturbances in general (17). The activity determined by the inflammation in areas such as the amygdala, hypothalamus, the insular cortex, and anterior cingulate cortex generates the perception of fatigue in MS patients (18). Based on these findings, there are complex mechanisms meant to clarify the appearance of fatigue in MS patients, therefore it should not be only seen as a consequence of the cardiovascular dysfunctions (17). Moreover, studies show no correlation between the severity of the disease and its duration, despite the popular belief $(11,17)$. For improving the perception of fatigue measures such as physical conditioning, the avoidance of cigarette smoking and improving sleep hygiene may be sufficient, while the most common pharmacological treatment includes Amantadine and Modafinil. Fatigue of MS patient and myalgic encephalomyelitis/chronic fatigue syndrome patients must be well differentiated, as long as the two entities have other common symptoms (19).

Thermoregulatory dysfunction is highly prevalent among MS patients, the neurological symptoms aggravating by environmental increases or decreases in core or only skin temperature. The increase in core body temperature with only $0.5^{\circ} \mathrm{C}$ may influence and worsen sensory, cognitive, and autonomic symptoms, known as Uhthoff's phenomenon. The mechanisms behind this phenomenon include changes in the axons affected by the demyelination and also in the ion channels due to the changes in the body temperature that result in diminished or blocked transmission through those fibers. Preeminently lesions in the brainstem rather than in the hypothalamus would be involved (3). Cold sensitivity is also causing worsening of symptoms among patients, with a higher proportion of them complaining about it during winter than during cold baths, demyelinating lesions in the hypothalamus being primarily involved (3).

Focussing on the symptoms that patients with heat sensitivity claim, they involve sensory, cognitive, and motor impairments. The cognitive deterioration implies a decline in sustained attention and concentration, slow reaction times, and an increase in error making. The most common sensory symptoms reported are temperature sensitivity, paraesthesia, and body pain. Motor impairments include fatigue, balance deficits, spasticity, muscle weakness, and dysarthria (3). Multiple studies that evaluated the thermoregulatory dysfunctions in MS are showing little evidence on whether only changes in core temperature are responsible for the symptoms or skin temperature is also involved. Recent observations are indicating that, in some cases, increasing the skin temperature with $3.8^{\circ} \mathrm{C}$ determines a decrease in postural stability and also an increase in motor symptoms (20). Immersion in hot water of both whole-body and upper or lower limb involved general body weakness, blurred vision, tachycardia, and loss of leg strength in patients. These findings are revealing that solely increasing the skin temperature may lead to triggering heat sensitivity (3). However, considering the increase in core temperature, studies are reporting perceptions of fatigue and also impairment in hand motor function in patients assessed, 
although skin temperature was also increased in this case. Hence, a combination of changes in both core and skin temperature in determining heat sensitivity may be implied $(3,21)$. Cold-induced sensitivity involves also sensory, cognitive, and motor impairments. Among sensory symptoms are also paraesthesia, altered body sensations, and vertigo sensation. Cognitive impairment includes language disturbances, attention deficits, and impaired memory processes, while motor impairments reported are body stiffness, spastic paraparesis, tremor, and ataxia in extremities and also blurred vision (3). Regarding cold sensitivity-induced impairments, studies show that decreases in core temperature are involved in slowing the neural transmission especially in patients with hypothalamic lesions, although some case studies report patients that are lacking those lesions (3). An important aspect concerning the decrease in body temperature in patients with cold sensitivity, although it may have a negative impact on some of them, body cooling is also beneficial in patients with heat sensitivity helping to decrease the core temperature and also ameliorating the symptoms. Studies report that whole body or forearm cold water immersion or barely cold water ingestion improve motor symptoms, back pain, and exercise tolerance, although the latter is not supported by all studies $(3,22)$. A study including 16 patients with MS shows no correlation between increased temperature and fatigue during exercise. One of the most useful and easiest methods in determining the thermoregulatory dysfunction is by measuring body temperature. To determine the thermoregulatory sweating response, as it seems to be affected in patients with autonomic dysfunction, thermoregulatory sweat test may be performed, but it cannot differentiate between central and peripheral lesions testing only the integrity of central pathways from the hypothalamus to the sweat glands.

\section{Conclusions}

Autonomic signs and symptoms of MS patients are frequently underdiagnosed and neglected in clinical practice. Their diagnosis has not entered into routine management. As important as the diagnosis is, the treatment and psychological support, lead to increased quality of life.

\section{Acknowledgements}

Not applicable.

\section{Funding}

No funding was received.

\section{Availability of data and materials}

Not applicable.

\section{Authors' contributions}

All the authors substantially contributed to each of the following aspects of this review paper: Design and conception (CAS, RMM, CFP and FIR); selection, analysis and interpretation of references (OGB, AMS and MCG); drafting of the manuscript (CAS, OGB, AMS and FIR); critical revision of the manuscript (CFP, OGB, MCG, FIR and RMM). All authors read and approved the final version of the manuscript.

\section{Ethics approval and consent to participate}

Not applicable.

\section{Patient consent for publication}

Not applicable.

\section{Competing interests}

The authors declare that they have no competing interests.

\section{References}

1. Racosta JM, Kimpinski K, Morrow SA and Kremenchutzky M: Autonomic dysfunction in multiple sclerosis. Auton Neurosci 193: $1-6,2015$

2. Lensch E and Jost WH: Autonomic disorders in multiple sclerosis. Autoimmune Dis 2011: 803841, 2011.

3. Christogianni A, Bibb R, Davis S, Jay O, Barnett M, Evangelou N and Filingeri D: Temperature sensitivity in multiple sclerosis: An overview of its impact on sensory and cognitive symptoms. Temperature (Austin) 5: 208-223, 2018.

4. Sternberg Z: Sympathetic nervous system dysfunction in multiple sclerosis, linking neurodegeneration to a reduced response to therapy. Curr Pharm Des 18: 1635-1644, 2012.

5. Mahajan ST, Patel PB and Marrie RA: Under treatment of overactive bladder symptoms in patients with multiple sclerosis: An ancillary analysis of the NARCOMS patient registry. J Urol 183: 1432-1437, 2010.

6. Bratu O, Mischianu D and Constantinoiu S: Transobturator urethral suspension surgical treatment of urinary incontinence in men. Chirurgia (Bucur) 108: 250-255, 2013.

7. Bratu O, Marcu D, Spinu D, Radulescu A, Oprea I and Mischianu D: TOT versus TVT-mesh surgical treatment in stress urinary incontinence. Rom J Mil Med 118: 40-44, 2015.

8. Spinu D, Bratu O, Marcu D, Mischianu D, Huica R, Surcel M, Munteanu A, Socea B, Bodean O and Ursaciuc C: The use of ELISA and PCR in identifying correlations between viral infections and benign prostatic hypertrophy. Rev Chim 69: 645-649, 2018.

9. Adamec I and Habek M: Autonomic dysfunction in multiple sclerosis. Clin Neurol Neurosurg 115 (Suppl 1): S73-S78, 2013.

10. Preziosi G, Raptis DA, Raeburn A, Thiruppathy K, Panicker J and Emmanuel A: Gut dysfunction in patients with multiple sclerosis and the role of spinal cord involvement in the disease. Eur J Gastroenterol Hepatol 25: 1044-1050, 2013.

11. Kanjwal K, Karabin B, Kanjwal Y and Grubb BP: Autonomic dysfunction presenting as postural orthostatic tachycardia syndrome in patients with multiple sclerosis. Int J Med Sci 7: 62-67, 2010.

12. Sirbu CA, Sirbu OM, Constantin C and Sandu AM: Neuroimmunotoxicity of aluminum. Farmacia 63: 8-10, 2015.

13. Lamtych M, Kubiak K, Kwiatkowska $\mathrm{K}$ and Badiuk N: Assessment of autonomic nervous system function in a patient suffering from multiple sclerosis-a case study. J Educ Health and Sport 8: 1039-1044, 2018.

14. Damla O, Altug C, Pinar KK, Alper K, Dilek IG and Kadriye A: Heart rate variability analysis in patients with multiple sclerosis. Mult Scler Relat Disord 24, 64-68, 2018.

15. Studer V, Rocchi C, Motta C, Lauretti B, Perugini J, Brambilla L, Pareja-Gutierrez L, Camera G, Barbieri FR, Marfia GA, et al: Heart rate variability is differentially altered in multiple sclerosis: Implications for acute, worsening and progressive disability. Mult Scler J Exp Transl Clin 3: 2055217317701317, 2017.

16. Hilz MJ: Cardiac stunning as first manifestation of multiple sclerosis: A case report reminding us not to overlook cardiovascular autonomic dysfunction in multiple sclerosis. Mult Scler 22: 847-848, 2016. 
17. Sander C, Hildebrandt H, Schlake HP, Eling P and Hanken K Subjective cognitive fatigue and autonomic abnormalities in multiple sclerosis patients. Front Neurol 8: 475, 2017.

18. Hanken K, Eling P and Hildebrandt H: The representation of inflammatory signals in the brain - a model for subjective fatigue in multiple sclerosis. Front Neurol 5: 264, 2014.

19. Strand EB, Nacul L, Mengshoel AM, Helland IB, Grabowski P, Krumina A, Alegre-Martin J, Efrim-Budisteanu M, Sekulic S, Pheby D, et al: Myalgic encephalomyelitis/chronic fatigue Syndrome (ME/CFS): Investigating care practices pointed out to disparities in diagnosis and treatment across European Union. PLoS One 14: e225995, 2019.
20. Poh PYS, Adams AN, Huang M, Allen DR, Davis SL, Tseng AS and Crandall CG: Increased postural sway in persons with multiple sclerosis during short-term exposure to warm ambient temperatures. Gait Posture 53: 230-235, 2017.

21. White AT, VanHaitsma TA, Vener J and Davis SL: Effect of passive whole body heating on central conduction and cortical excitability in multiple sclerosis patients and healthy controls. J Appl Physiol (1985) 114: 1697-1704, 2013.

22. Chaseling GK, Filingeri D, Barnett M, Hoang P, Davis SL and Jay O: Cold water ingestion improves exercise tolerance of heat-sensitive people with MS. Med Sci Sports Exerc 50: 643-648, 2018. 